\title{
Surveillance du patient sous ventilation mécanique invasive
}

\author{
Monitoring of invasive mechanical ventilation
}

\section{A. Constan - I. Bourgeon-Ghittori - F. Schortgen - Service de réanimation médicale, Créteil}

Reçu le 5 octobre 2011 ; accepté le 6 décembre 2011

(C) SRLF et Springer-Verlag France 2011

Résumé La ventilation mécanique invasive a pour but de maintenir ou de restaurer l'hématose en cas d'incapacité ventilatoire totale ou partielle. Il s'agit d'une technique spécifique et complexe dont les mécanismes doivent être compris. La surveillance du patient ventilé a pour objectifs d'évaluer l'efficacité du support ventilatoire, de prévenir et de détecter précocement la survenue de complications, de diminuer la durée de la ventilation et d'optimiser le confort du patient. Étant au plus proche du patient, l'infirmier(ière) est en première ligne dans la surveillance de l'efficacité et de la tolérance de cette technique. Cet article propose une mise au point sur les éléments de surveillance d'un patient sous ventilation mécanique invasive basée sur des critères spécifiques relevant de l'examen clinique pulmonaire et extrapulmonaire, du mode ventilatoire, de la gestion des alarmes mais également du confort du patient et de son adaptation au ventilateur. Pour citer cette revue : Réanimation 21 (2012).

Mots clés Ventilation mécanique · Surveillance infirmière · Interaction patient-ventilateur $\cdot$ Confort $\cdot$ Complication

Abstract Invasive mechanical ventilation allows total or
partial respiratory support and restores adequate blood gas
exchanges. This complex technique needs specific know-
ledge to improve management. The objectives of monitoring
mechanical ventilation include prevention and early detec-
tion of complications, decrease in mechanical ventilation

A. Constan $(\triangle)$

Réseau REVA, service de réanimation médicale,

AP-HP, groupe hospitalier Henri Mondor,

51 avenue du Maréchal-de-Lattre-de-Tassigny,

F-94010 Créteil cedex,

France http://revaweb.org

e-mail : adrien.constan@hmn.aphp.fr

A. Constan · I. Bourgeon-Ghittori · F. Schortgen

Service de réanimation médicale,

AP-HP, groupe hospitalier Henri-Mondor,

51, avenue du Maréchal-de-Lattre-de-Tassigny,

F-94010 Créteil cedex, France duration, and improvement of patient's comfort. Nurses are particularly involved in the evaluation of the efficiency of mechanical ventilation and the detection of its complications. In this article, we propose an update on the monitoring of the patient under invasive mechanical ventilation based on clinical examination, information from the ventilator, alarms management, and patient-ventilator interaction. To cite this journal: Réanimation 21 (2012).

Keywords Mechanical ventilation - Nurse monitoring · Patient-ventilator interaction · Adverse effects · Comfort

\section{Introduction}

La ventilation mécanique invasive est une technique fondamentale en réanimation. Son but est d'assurer une hématose correcte. La mise en œuvre de cette technique impose le recours à un appareillage complexe (le ventilateur) et à des dispositifs invasifs (sonde d'intubation, canule de trachéotomie) qui peuvent être source de complications graves. La surveillance du patient ventilé a pour objectifs d'évaluer l'efficacité de la technique, de prévenir et de détecter précocement la survenue de complications, de diminuer la durée de la ventilation et d'optimiser le confort du patient. Les éléments de surveillance d'un patient sous ventilation mécanique invasive comportent des critères spécifiques relevant de l'examen clinique pulmonaire et extrapulmonaire, du mode ventilatoire, de la gestion des alarmes mais également $\mathrm{du}$ confort du patient et de son adaptation au ventilateur.

\section{Surveillance clinique de la ventilation}

\section{Signes respiratoires}

L'infirmier(ière) est garant de l'intégrité et du bon fonctionnement du matériel nécessaire à la ventilation. La présence de fuites ou d'obstacles sur le circuit ventilatoire induit une mauvaise qualité de la ventilation et peut s'accompagner de signes de détresse respiratoire. L'hypoventilation expose le 
patient à l'hypoxie, et potentiellement à l'arrêt cardiaque. Les fuites peuvent être présentes en cas de déconnexion d'un élément du circuit ventilatoire (câbles de l'humidificateur chauffant, obturateur du raccord annelé, branche inspiratoire, système d'aérosolisation) ou de perte de pression du ballonnet de la sonde. L'infirmier(ière) doit donc s'assurer du montage du circuit selon les recommandations du constructeur et vérifier la pression du ballonnet.

L'observation du patient est primordiale pour dépister toute complication ou mauvaise tolérance de la ventilation. Une ventilation bien tolérée est caractérisée par une respiration calme et régulière, une amplitude symétrique du thorax, un faciès détendu. La persistance ou l'apparition de signes de détresse respiratoire comme la cyanose, les sueurs, le tirage sus-sternal ou le balancement thoracoabdominal peuvent témoigner d'une assistance respiratoire insuffisante ou de l'apparition d'une complication. Ils doivent faire rechercher des causes d'hypoxie, d'hypercapnie ou une désadaptation au mode ventilatoire.

La surveillance régulière de la pression du ballonnet est nécessaire afin de la maintenir dans les valeurs recommandées. Étant donné la variation de la pression du ballonnet en fonction des événements (toux, mobilisation, aspiration trachéale, sédation), certaines équipes proposent même son monitorage continu [1]. La pression du ballonnet doit toujours être comprise entre 20 et $30 \mathrm{cmH}_{2} \mathrm{O}[2,3]$. Une pression du ballonnet supérieure à ces valeurs expose le patient à des complications trachéales à type d'ischémie, de sténose ou de fistule œsotrachéale [4]. Une pression inférieure à ces valeurs expose le patient à un risque de fuites et d'inhalation des sécrétions sus-glottiques.

L'occlusion de la sonde d'intubation peut être provoquée par un bouchon muqueux, la morsure de la sonde par le patient, un mauvais positionnement de la sonde sur la carène ou la paroi trachéale et la plicature de la sonde en cas d'utilisation d'un petit diamètre.

La sonde d'intubation doit en permanence être fixée de manière efficace. Plusieurs procédures peuvent être utilisées, aucune n'ayant été clairement évaluée. La fixation doit empêcher les mouvements de la sonde et permettre d'éviter une extubation accidentelle ou une intubation sélective. Pour s'assurer du bon positionnement de la sonde, l'infirmier (ière) vérifie régulièrement le repère gradué au niveau de l'arcade dentaire, particulièrement avant mobilisation du patient. Ce repère est noté sur la feuille de surveillance quotidienne, il ne peut être modifié que sur prescription médicale. L'infirmier peut ausculter chaque poumon à l'aide du stéthoscope afin de s'assurer de l'homogénéité de la ventilation. La peau doit être l'objet d'une surveillance attentive sur tout le trajet de la fixation. Une fixation humide ou souillée favorise l'apparition ou l'aggravation des lésions cutanées induites par cette fixation.

\section{Surveillance clinique des effets extrarespiratoires de la ventilation}

\section{Signes cardiovasculaires}

En ventilation spontanée (VS), la contraction diaphragmatique pendant l'inspiration induit une pression intrathoracique négative qui permet la mobilisation du volume courant $(\mathrm{Vt})$. Cette pression négative facilite le retour du sang présent dans les veines caves vers le cœur. Cela permet au cœur de disposer d'un volume de sang plus important lors de ses contractions (augmentation de la précharge) et donc d'augmenter son débit. En ventilation mécanique sur sonde, la pression intrathoracique est positive, au moins lors de l'inspiration, et constitue alors une gêne au retour veineux. La sédation et l'hypovolémie peuvent majorer la diminution du retour veineux et exposent le patient à une baisse du débit cardiaque [5]. Le patient ventilé est donc particulièrement exposé aux complications hémodynamiques, et justifie d'une surveillance rapprochée des constantes hémodynamiques. Chez les patients avec une dysfonction du ventricule gauche, la ventilation en pression positive peut, cependant, être bénéfique en diminuant le travail cardiaque lors de l'éjection systolique du fait d'un gradient de pression plus important entre le thorax et l'aorte extrathoracique (baisse de la postcharge). Le retour à un régime de pressions thoraciques négatives qui accompagne les tests de sevrage de la ventilation implique une majoration du travail ventriculaire gauche et favorise l'apparition d'un œdème pulmonaire [6]. Cette situation expose le patient à des difficultés de sevrage.

Le maintien de l'équilibre entre les apports et les pertes liquidiennes, objectivé par le bilan entrée-sortie quotidien, permet de prévenir une surcharge hydrique excessive qui peut majorer l'œdème pulmonaire et l'hypoxémie lorsqu'il existe une lésion pulmonaire inflammatoire de type syndrome de détresse respiratoire aiguë (SDRA) ou d'augmenter le risque d'œdème pulmonaire cardiogénique lors du retour à une VS au cours du sevrage.

\section{Signes neurologiques}

L'apparition de signes neurologiques allant de l'agitation au coma peut témoigner de la mauvaise tolérance de la ventilation ou d'une dégradation respiratoire responsable d'une hypoxie et/ou d'une hypercapnie. L'infirmier(ière) doit donc être vigilant à l'apparition ou à l'aggravation de troubles de la conscience et toujours chercher à en connaître la cause précise [7].

\section{Signes digestifs}

L'alitement, l'administration de morphiniques, la pudeur sont des facteurs favorisant le ralentissement du transit normal. Les 
patients sont donc exposés, d'une part, au risque de vomissements et d'inhalation et, d'autre part, au risque d'une distension abdominale pouvant gêner les mouvements diaphragmatiques et augmenter les pressions intra-abdominales et thoraciques. Cette distension peut être à l'origine de troubles ventilatoires majeurs. Le transit du patient intubé ventilé doit donc faire l'objet d'une surveillance. La présence de la sonde gastrique peut également favoriser les reflux gastriques [8].

\section{Signes infectieux}

La pneumonie acquise en ventilation mécanique (PAVM) est la première cause d'infection acquise en réanimation, elle doit donc être prévenue et dépistée rapidement afin de débuter un traitement approprié.

La position du patient est extrêmement importante dans la prévention des PAVM. En dehors de contre-indications (patient avec lésion du rachis, instabilité hémodynamique grave), un patient intubé-ventilé doit être mis en position demi-assise (minimum à $30^{\circ}$ ) [2]. Cette position limite le reflux gastrique vers la trachée et diminue l'incidence des PAVM $[8,9]$. Le contrôle régulier de la pression du ballonnet de la sonde, la lutte contre les régurgitations et les soins antiseptiques de la bouche et des voies aériennes supérieures sont les principales autres mesures proposées pour limiter le risque et les conséquences d'une inhalation [2]. L'intensité de la sédation a également un impact direct sur la durée de ventilation mécanique et donc l'incidence des PAVM [10,11]. La surveillance du niveau de sédation doit être faite à intervalles réguliers selon une procédure de service, au mieux à l'aide d'un score [7].

La fièvre, l'aspect purulent et l'abondance des sécrétions trachéales font partie des signes cliniques d'infection pulmonaire. Leur surveillance doit donc aider au diagnostic clinique précoce qui sera confirmé par un prélèvement bactériologique des sécrétions pulmonaires. En cas de SDRA, les éléments cliniques et radiologiques du diagnostic de PAVM sont moins spécifiques. Il reste cependant nécessaire de traiter le plus précocement possible une PAVM chez ces patients déjà très hypoxémiques.

\section{Signes neurolocomoteurs}

Le patient ventilé doit être mobilisé le plus précocement possible afin de limiter ou prévenir la survenue de complications liées au décubitus prolongé (fonte musculaire, escarres, rétractions, ankyloses, hypotension orthostatique, ralentissement du transit intestinal). La possibilité d'asseoir le patient au bord du lit, de le mettre au fauteuil, de le lever avec appui, ou de le faire marcher doit être recherchée le plus tôt possible $[12,13]$. La mobilisation précoce aurait un impact positif sur le devenir du patient avec un retour plus rapide à l'autonomie (toilette, marche sans aide, habillage), une durée plus courte du delirium de réanimation, et un raccourcissement de la durée d'hospitalisation [14].

\section{Surveillance paraclinique}

\section{Monitorage et examens complémentaires}

La surveillance continue de la saturation pulsée en oxygène $\left(\mathrm{SpO}_{2}\right)$ est systématique chez le patient ventilé en réanimation. Elle reflète l'efficacité des échanges gazeux. Toutefois, la fiabilité de la valeur affichée par l'oxymétrie de pouls varie en fonction de la perfusion périphérique (bas débit cardiaque, état de choc, perfusion de vasopresseurs) et des per-

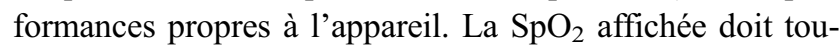
jours être confrontée à des éléments cliniques et en cas de doute sur sa fiabilité, elle doit être comparée à la saturation artérielle en oxygène $\left(\mathrm{SaO}_{2}\right)$ par la réalisation d'un gaz du sang.

Les objectifs de $\mathrm{SpO}_{2}$ peuvent varier en fonction de la pathologie du patient et devraient être prescrits afin de faciliter la surveillance. Par exemple, au cours du SDRA, l'objectif habituel est une $\mathrm{SpO}_{2}$ entre 88 et $95 \%$ afin de limiter l'exposition à une fraction inspirée en oxygène $\left(\mathrm{FiO}_{2}\right)$ élevée, potentiellement toxique pour le poumon. Lors de la ventilation d'un patient ayant une bronchopathie chronique obstructive (BPCO), l'objectif peut être de 88-92\% afin d'éviter une majoration de l'hypercapnie par une oxygénation trop importante.

Le taux de dioxyde de carbone expiré $\left(\mathrm{EtCO}_{2}\right)$, mesuré par la capnographie, est un reflet indirect de la pression en dioxyde de carbone dans le sang artériel $\left(\mathrm{PaCO}_{2}\right)$. Sa valeur normale se situe entre 35 et $45 \mathrm{mmHg}$. En cas de lésion pulmonaire et/ou d'état de choc, elle n'est plus un reflet fiable de la $\mathrm{PaCO}_{2}$. Son utilisation est recommandée au cours de l'intubation pour s'assurer du positionnement de la sonde dans la trachée. En revanche, un monitorage continu de la capnographie n'est pas recommandé chez tous les patients. Cependant, la capnographie peut être utilisée dans certaines situations ou une évaluation régulière de la $\mathrm{PaCO}_{2}$ est recommandée, notamment chez le patient traumatisé crânien.

La fréquence des prélèvements pour analyse des gaz du sang dépend de l'affection et de l'état du patient. Certaines modifications des réglages du ventilateur peuvent avoir un impact significatif sur l'oxygénation (pression expiratoire positive [PEP], $\mathrm{FiO}_{2}$ ). Leur retentissement sur l'hématose devrait être contrôlé par la gazométrie plutôt que la simple surveillance de la $\mathrm{SpO}_{2}$ [15]. De la même manière, tout doute sur une valeur de $\mathrm{SpO}_{2}$ aberrante devrait amener à prélever des gaz du sang. Pour connaître la fiabilité de la $\mathrm{SpO}_{2}$, la valeur indiquée par le scope doit être comparée à la $\mathrm{SaO}_{2}$ des gaz du sang. Toutefois, l'écart observé n'est pas 
reproductible, et il ne permet pas d'extrapoler une corrélation entre ces deux valeurs.

La ventilation invasive ne devrait plus être une raison suffisante pour réaliser une radiographie de thorax systématique quotidienne. Il a en effet été montré qu'une politique de radiographie « à la demande », en fonction d'une évaluation quotidienne des médecins et de l'apparition d'incidents, permettait de réduire le nombre de clichés effectués sans nuire à l'évolution des patients [16].

\section{Surveillance de la fonction ventilatoire à l'aide du ventilateur}

L'évaluation de la fonction ventilatoire, de l'adaptation du patient et de la survenue potentielle de complications varie en fonction du mode ventilatoire utilisé. Pour cela, il est nécessaire que l'infirmier connaisse les principes de base du fonctionnement du mode ventilatoire appliqué à son patient.

\section{Mode ventilatoire en volume : la ventilation assistée contrôlée (VAC)}

En ventilation en volume contrôlé, le support ventilatoire est totalement assuré par le ventilateur. Les paramètres réglés sont : la fréquence respiratoire (FR), le Vt, la PEP, la $\mathrm{FiO}_{2}$ et le débit inspiratoire (ou le temps inspiratoire). Le Vt est le volume de gaz insufflé par le ventilateur à chaque cycle respiratoire. La valeur usuelle se situe entre 6 et $10 \mathrm{ml} / \mathrm{kg}$ de poids prédit par le sexe et la taille. Le débit inspiratoire (en litre par minute) correspond à la vitesse par unité de temps à laquelle le Vt est insufflé dans les voies aériennes. Pour un même $\mathrm{Vt}$, plus le débit est élevé, plus le temps inspiratoire est court (temps inspiratoire $=\mathrm{Vt} / \mathrm{débit})$. Le débit est généralement réglé aux alentours de $60 \mathrm{l} / \mathrm{min}$. Le débit inspiratoire a un impact sur le travail respiratoire et le confort du patient. Il peut donc être augmenté ou réduit afin d'optimiser le confort. La FR est généralement réglée entre 15 et 30 cycles par minute. La variable principale qui détermine le réglage de la FR est la $\mathrm{PaCO}_{2}$.

La VAC se distingue de la ventilation contrôlée (VC) par un réglage supplémentaire, le trigger de la valve inspiratoire. La présence du trigger offre au patient la possibilité de déclencher des cycles respiratoires. Plus le trigger est sensible, plus l'inspiration sera déclenchée facilement par le patient. En VAC, la FR réglée représente la FR minimale, le patient pouvant déclencher des cycles supplémentaires. Il est possible que le patient déclenche la totalité des cycles et qu'il n'y ait aucun cycle contrôlé. Toutefois, le patient ne sera responsable que de l'initiation des cycles. Le Vt et le débit inspiratoire (et donc le temps inspiratoire) resteront identiques que le cycle soit assisté (déclenchement patient) ou contrôlé (déclenchement machine). En VAC, il est donc utile d'évaluer la proportion « cycles assistés/cycles contrô- lés » qui reflète l'autonomie respiratoire du patient et la possibilité de passer en VS avec aide inspiratoire (VSai) si les cycles assistés sont plus fréquents que les cycles contrôlés. Cette donnée est accessible sur la plupart des ventilateurs modernes. L'effort du patient qui déclenche l'inspiration est également visible sur la courbe de pression, il se traduit par une dépression minime de la courbe de pression immédiatement avant le début du cycle.

En VC/VAC, le Vt est invariable puisqu'il est réglé, ce sont les pressions qui varient en cas de modification de la mécanique ventilatoire du patient. Les paramètres à surveiller plus particulièrement sont donc la pression de crête (Pcrête ou Pmax) et la pression de Plateau (Pplat).

La Pcrête est la pression maximale qui règne dans les voies aériennes en fin d'inspiration. La Pcrête est mesurée au $\langle\mathrm{Y} »$ du circuit ventilatoire et diminue tout au long de l'arbre bronchique du fait des résistances des voies aériennes. Elle n'atteint donc pas les alvéoles. Cette pression dépasse rarement $50 \mathrm{cmH}_{2} \mathrm{O}$. La pression qui reflète au mieux la pression qui règne au niveau alvéolaire est la Pplat qui, elle, ne dépend pas des résistances des voies aériennes. C'est une pression mesurée lors d'un arrêt du débit au cours de l'inspiration qui permet l'annulation des pressions résistives. La pression statique alors mesurée ne reflète que les pressions élastiques du système respiratoire. La Pplat ne doit pas dépasser 28 à $30 \mathrm{cmH}_{2} \mathrm{O}$, notamment chez le patient en SDRA [17]. En pratique, la Pplat peut être mesurée de deux manières différentes : lors d'une pause téléinspiratoire manuelle effectuée par une manipulation du respirateur ou lors d'une pause téléinspiratoire d'au moins 0,2 seconde programmée sur chaque cycle (temps de plateau). La deuxième méthode permet l'affichage continu de la Pplat par le ventilateur, ce qui facilite sa surveillance. La surveillance de ces pressions a pour but de détecter une valeur excessive afin d'éviter la survenue de lésions pulmonaires par barotraumatismes. Elle permet aussi de détecter une modification de la mécanique ventilatoire du patient.

Les modifications de pression liées à la mécanique ventilatoire peuvent être comprises en s'appuyant sur l'équation du mouvement du système respiratoire.

Équation $1:$ Pression des voies aériennes $=\mathrm{Po}+$ pression résistive + pression élastique, où Po est la pression dans les voies aériennes avant l'inspiration, avec pression résistive $=($ résistances $\times$ débit $)$ et pression élastique $=(\mathrm{Vt} / \mathrm{com}-$ pliance). Cette équation met en évidence les propriétés mécaniques du système respiratoire reflétées par la pression élastique et la pression résistive.

La pression résistive dépend de deux facteurs : le débit inspiratoire, réglé par le médecin, et les résistances pulmonaires, qui correspondent à l'ensemble des forces qui s'opposent à l'écoulement des gaz dans les voies aériennes. Si le débit ou les résistances pulmonaires augmentent, les pressions résistives augmentent. La pression inspiratoire liée 


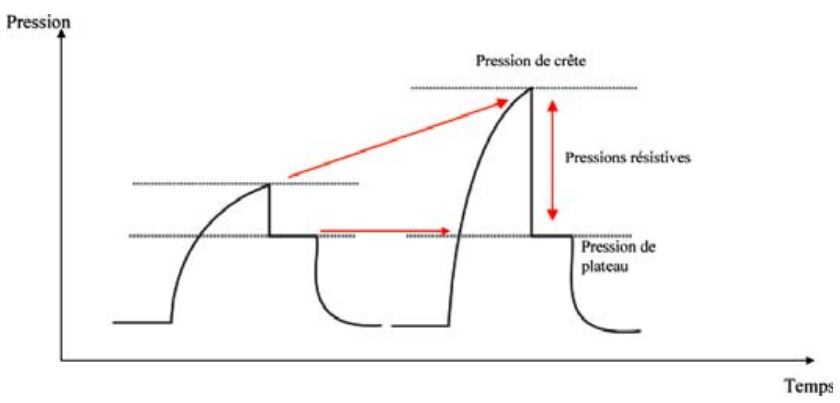

Fig. 1 L'augmentation des résistances à l'écoulement des gaz dans les voies aériennes entraîne une augmentation de la pression de crête, sans variation de la pression de plateau

aux résistances est reflétée sur le ventilateur par la différence entre la Pplat et la Pcrête (Fig. 1). Toute force s'opposant à l'écoulement du gaz augmente la pression résistive et se traduit par une augmentation de la Pcrête sans augmentation de la Pplat (Fig. 1). L'augmentation de la pression résistive (Pcrête-Pplat) fait suspecter :

- une obstruction de la sonde d'intubation (sécrétions, morsure du patient, pliure ou déplacement de la sonde sur la carène...);

- une obstruction des voies aériennes (lésion bronchique, caillots obstructifs, présence d'œdème ou de secrétions...);

- un bronchospasme.

La Pcrête est égale à l'addition de la pression résistive et de la Pplat. Si la Pplat augmente, la Pcrête augmentera donc de la même valeur. Dans cette situation, l'augmentation de la Pcrête n'est pas liée à une augmentation de la pression résistive (par augmentation des résistances dans les voies aériennes), mais à celle de la pression élastique (par baisse de la compliance pulmonaire)

La pression élastique dépend de deux facteurs : le Vt et la compliance pulmonaire. La compliance pulmonaire est la capacité du poumon à augmenter son volume en fonction

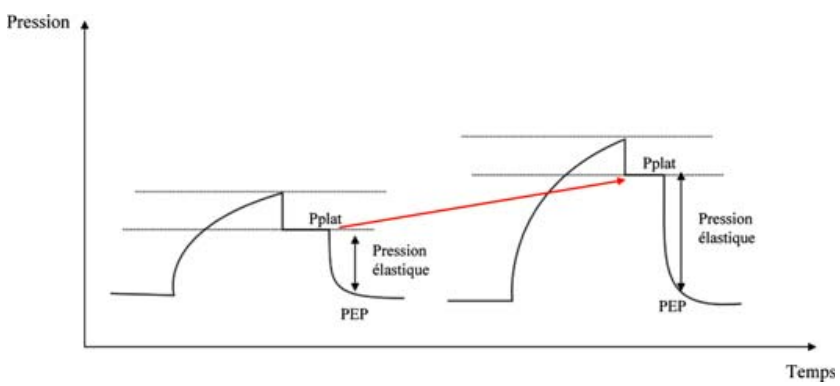

Fig. 2 Une diminution de la compliance thoracopulmonaire est responsable d'une augmentation de la pression de plateau (Pplat) et donc du risque barotraumatique. La pression de crête augmente en parallèle PEP : pression expiratoire positive d'une variation de pression. Plus la compliance est basse, plus la pression sera élevée pour un même volume. La compliance pulmonaire diminue si le volume accessible à la ventilation diminue. La pression élastique est reflétée sur le ventilateur par la différence entre la PEP et la Pplat (Fig. 2). Si le Vt est augmenté ou si la compliance diminue, la pression élastique (Pplat-PEP) sera plus élevée. Une baisse de la compliance est habituelle dans les situations suivantes :

- un pneumothorax, un épanchement pleural ou un hémothorax ;

- une atélectasie ;

- une intubation sélective ;

- une distension abdominale ;

- un comblement liquidien alvéolaire par un œedème, du pus ou du sang.

\section{Modes ventilatoires en pression}

\section{VSai}

En VSai, chaque cycle est déclenché par le patient, il n'y a pas de FR minimum. Les paramètres réglés sont : la pression d'aide inspiratoire, le trigger, la $\mathrm{PEP}$ et la $\mathrm{FiO}_{2}$.

La pression d'aide inspiratoire correspond au niveau de pression que le ventilateur insuffle dans les voies aériennes tout au long du temps inspiratoire. Ce niveau de pression ne peut pas varier au cours de l'inspiration, puisqu'il est réglé. Ainsi, quand le patient déclenche un cycle, le rôle du ventilateur est d'atteindre puis de maintenir pendant toute l'inspiration la pression au niveau de la pression d'aide réglée. Le niveau d'aide est réglé en fonction du Vt cible souhaité. Il est généralement compris entre 8 et $20 \mathrm{cmH}_{2} \mathrm{O}$. Un niveau d'aide minimum de $7 \mathrm{cmH}_{2} \mathrm{O}$ est requis pour compenser les pressions résistives d'un circuit avec humidificateur chauffant. Un niveau d'aide minimum de $10 \mathrm{cmH}_{2} \mathrm{O}$ est requis pour compenser les pressions résistives d'un circuit avec filtre échangeur de chaleur et d'humidité. L'augmentation du niveau d'aide doit permettre la diminution du travail respiratoire. Celle-ci se traduira par une diminution de la FR associée à l'augmentation du Vt.

Le Vt peut varier sans modification du niveau de pression d'aide inspiratoire. En effet, si le patient fait un effort plus important, la dépression thoracique sera plus grande, la pression de départ sera donc plus basse, et le ventilateur délivrera plus de volume pour atteindre la pression d'aide inspiratoire réglée. Le $\mathrm{Vt}$ peut également varier en cas de modifications de la mécanique ventilatoire. Une augmentation des résistances dans les voies aériennes ou une baisse de la compliance entraînent une baisse du Vt. Le Vt est donc un paramètre à surveiller tout particulièrement en VSai. 


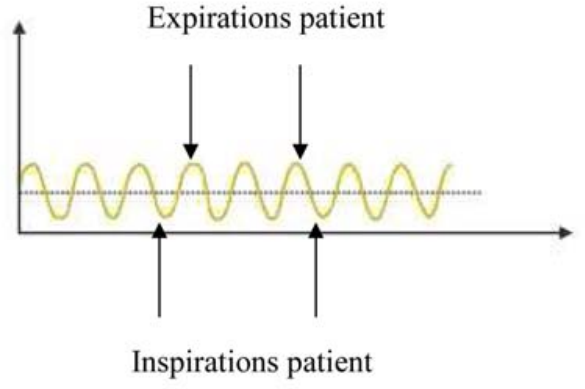

Ventilation spontanée à niveau de pression positive unique (CPAP)

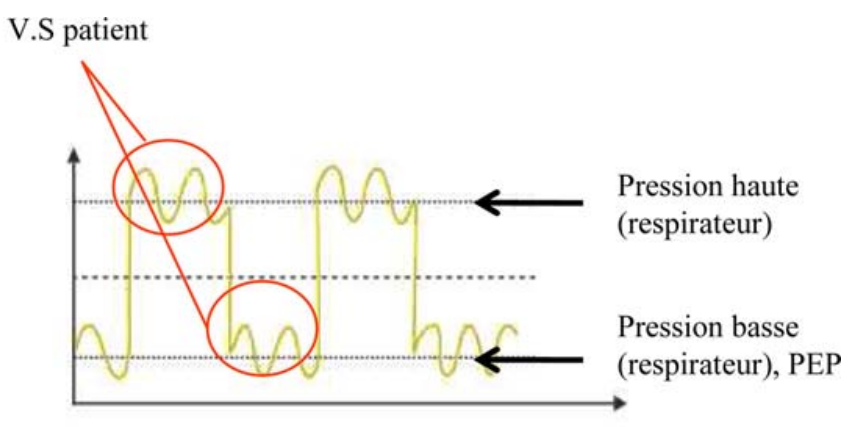

BiPAP

Fig. 3 La BiPAP ou bi-level positive airway pressure associe deux niveaux de pression régulés par la machine, inspiratoire et expiratoire, à une ventilation spontanée (VS) qui s'applique à chacun des deux niveaux CPAP : VS en pression positive ; PEP : pression expiratoire positive

- Biphasic positive airway pressure (BiPAP)-airway pressure release ventilation (APRV)

Le principe de ce mode est d'associer une alternance fixe et régulière de pressions (pression haute et pression basse correspondant à la PEP) tout en laissant les valves du ventilateur ouvertes (BiPAP), permettant ainsi au patient de respirer à tout moment que ce soit sur la pression haute ou la pression basse (Fig. 3) [18]. En fonction des différents modes en pression et des différentes marques de ventilateurs, les cycles déclenchés par le patient seront, avec ou sans assistance, délivrés par le ventilateur. Les réglages sont : un niveau de pression haute, un temps de pression haute, la pression basse correspondant à la PEP et le temps de pression basse et la $\mathrm{FiO}_{2}$. Ainsi, le ventilateur alterne une pression haute pendant le temps de pression haute réglé (temps inspiratoire) et une PEP pendant le temps de pression basse réglé (temps expiratoire). Ces quatre éléments sont invariables. Un des intérêts de ces modes ventilatoires est de préserver la VS du patient et éviter l'atrophie rapide du diaphragme.

Les paramètres à surveiller sont : la FR patient, le volumeminute patient et volume-minute machine. Il est utile de dissocier la part de ventilation imputable à la machine et celle imputable au patient afin de détecter la présence d'une VS mais de vérifier également qu'elle ne soit pas trop importante en cas de lésion pulmonaire sévère de type SDRA.

Comme en VSai, au cours de la BiPAP-APRV, l'augmentation des pressions résistives et la diminution de la compliance pulmonaire se traduisent toutes deux par une diminution du Vt sans modification de la Pcrête qui est déterminée par le niveau de pression haute ou d'aide inspiratoire réglée. Si les résistances sont plus élevées, la pression réglée est atteinte plus rapidement, le temps inspiratoire est plus court, et le Vt plus bas. Si la compliance diminue, par définition, le volume sera plus faible pour une même pression. Ces modes ne permettent donc pas de différencier des modifications de pressions élastiques et/ou de pressions résistives.

\section{Surveillance des alarmes du ventilateur}

Les limites d'alarmes sont en général réglées avec une fourchette de 20 à $30 \%$ en dessous et au-dessus de la valeur de base du patient. L'infirmier(ière) doit vérifier leur réglage à chaque prise de poste ainsi qu'à chaque changement de mode ventilatoire. La modification ponctuelle du mode ou d'un paramètre ventilatoire doit amener à vérifier les seuils d'alarmes. Les paramètres à surveiller et les principales sources d'alarme peuvent varier en fonction du mode ventilatoire et sont résumés dans le Tableau 1.

\section{Adaptation patient-ventilateur}

Les asynchronies sont fréquentes au cours de la ventilation mécanique et peuvent avoir pour conséquences l'inconfort, l'augmentation de la durée de la VM et du séjour en réanimation, l'altération de la qualité du sommeil et un sevrage ventilatoire plus difficile [19-22]. Il est donc important de pouvoir les dépister pour y remédier. L'infirmier étant le soignant le plus en contact avec le patient, il peut jouer un rôle de premier ordre dans la détection des asynchronies. Les trois principaux types d'asynchronies sont les efforts inefficaces, les double-déclenchements et les autodéclenchements.

Les efforts inefficaces sont des efforts inspiratoires non suivis du déclenchement d'un cycle respiratoire. Ils se traduisent par une inflexion sur la courbe de débit expiratoire (Fig. 4). Les causes principales sont un effort insuffisant, un 
Tableau 1 Principales causes d'alarmes au cours de la ventilation mécanique

\begin{tabular}{|c|c|c|}
\hline \multirow[t]{2}{*}{ Alarme } & \multicolumn{2}{|l|}{ Causes possibles } \\
\hline & Modes en pression (VSai/BiPAP-APRV) & Mode assisté contrôlé (VAC) \\
\hline $\begin{array}{l}\text { Pcrête haute } \\
\text { ou en augmentation } \\
\left(>50 \mathrm{cmH}_{2} \mathrm{O}\right)\end{array}$ & - Efforts de toux & $\begin{array}{l}\text { - Efforts de toux } \\
\text { - Obstacles dans les voies aériennes : } \\
\text { sécrétions bronchiques, morsure } \\
\text { de la sonde, plicature de la sonde, } \\
\text { obstruction de sonde, bronchospasme } \\
\text { - Augmentation de Pplat }\end{array}$ \\
\hline Pcrête basse & - Fuites & $\begin{array}{l}\text { - Fuites } \\
\text { - Efforts inspiratoires importants } \\
\text { avec demande ventilatoire supérieure } \\
\text { à la ventilation délivrée (réveil, } \\
\text { désadaptation) }\end{array}$ \\
\hline $\begin{array}{l}\text { Pplat haute } \\
\left(>30 \mathrm{cmH}_{2} \mathrm{O}\right) \\
\text { ou en augmentation }\end{array}$ & - Pplat non mesurable par le ventilateur en ventilation spontanée & $\begin{array}{l}\text { - Diminution de la compliance } \\
\text { pulmonaire : intubation sélective, } \\
\text { SDRA, pneumothorax, épanchement } \\
\text { pleural, œdème pulmonaire, } \\
\text { atélectasie... } \\
\text { - Augmentation de l'auto-PEP } \\
\text { - Désadaptation du patient } \\
\text { avec expiration pendant la phase } \\
\text { de plateau }\end{array}$ \\
\hline FR élevée (> 35/min) & $\begin{array}{l}\text { - Augmentation de la demande ventilatoire : réveil, douleur, } \\
\text { agitation, fièvre, choc, hypoxémie, acidose } \\
\text { - Trigger trop sensible avec autodéclenchements } \\
\text { VSai } \\
\text { BiPAP-APRV } \\
\text { Pression d'aide insuffisante } \\
\end{array}$ & $\begin{array}{l}\text { - Augmentation de la demande } \\
\text { ventilatoire : réveil, douleur, agitation, } \\
\text { fièvre, choc, hypoxémie, acidose } \\
\text { - Volume courant réglé trop bas } \\
\text { - Trigger trop sensible } \\
\text { avec autodéclenchements }\end{array}$ \\
\hline Vti élevé (> 600 ml) & $\begin{array}{l}\text { Augmentation de la demande ventilatoire : réveil, douleur, } \\
\text { agitation, fièvre, choc, hypoxémie, acidose } \\
\text { VSai } \\
\text { BiPAP-APRV } \\
\text { Pression d'aide top élevée } \\
\begin{array}{c}\text { Pression haute trop } \\
\text { élevée et/ou Ti trop long }\end{array}\end{array}$ & - Vti réglé sur le ventilateur \\
\hline VE haute $(>151 / \mathrm{min})$ & - Mêmes causes possibles que FR élevée et/ou Vti élevé & $\begin{array}{l}\text { - Augmentation de la demande } \\
\text { ventilatoire : réveil, douleur, agitation, } \\
\text { fièvre, choc, hypoxémie, acidose } \\
\text { - FR élevée }\end{array}$ \\
\hline $\begin{array}{l}\text { FR basse }(<15 / \mathrm{min}) \\
\text { Apnée }\end{array}$ & $\begin{array}{l}\text { BiPAP-APRV } \\
\text { - Le Ti et Te réglés sur le ventilateur déterminent une FR } \\
\text { minimale } \\
\text { VSai } \\
\text { - Trouble de la commande ventilatoire : médicaments sédatifs, } \\
\text { morphiniques, pathologies neurologiques centrales } \\
\text { - Niveau d'aide trop élevé avec surassistance ventilatoire, } \\
\text { alcalose } \\
\text { - Efforts inefficaces non enregistrés par le ventilateur : trigger pas } \\
\text { assez sensible, auto-PEP, syndrome d'apnée du sommeil, force } \\
\text { musculaire insuffisante } \\
\text { - Maladies neuromusculaires } \\
\text { - Épuisement respiratoire }\end{array}$ & - FR minimale réglée sur le ventilateur \\
\hline
\end{tabular}




\begin{tabular}{|c|c|c|}
\hline \multirow[t]{2}{*}{ Alarme } & \multicolumn{2}{|l|}{ Causes possibles } \\
\hline & Modes en pression (VSai/BiPAP-APRV) & Mode assisté contrôlé (VAC) \\
\hline Vte bas $(<300 \mathrm{ml})$ & $\begin{array}{l}\text { - Épuisement respiratoire } \\
\text { - Alcalose } \\
\text { - Trouble de la commande ventilatoire : médicaments sédatifs, } \\
\text { morphiniques, pathologies neurologiques centrales } \\
\text { - Obstacles dans les voies aériennes : sécrétions bronchiques, } \\
\text { morsure de la sonde, plicature de la sonde, obstruction de sonde, } \\
\text { bronchospasme... } \\
\text { - Diminution de la compliance pulmonaire : intubation sélective, } \\
\text { SDRA, pneumothorax, épanchement pleural, œdème } \\
\text { pulmonaire, atélectasie... } \\
\text { - Fuites }\end{array}$ & - Fuites \\
\hline & $\begin{array}{cc}\text { VSai } & \text { BiPAP-APRV } \\
\text { Niveau d'aide insuffisant } & \text { Pression haute } \\
& \text { et/ou Ti insuffisant }\end{array}$ & \\
\hline VE basse $(<5$ 1/min $)$ & - Même causes possibles que FR basse et/ou Vte bas & - Fuites \\
\hline Fuites (Vte $<$ Vti) & $\begin{array}{l}\text { - Déconnexion d'un élément du circuit ventilatoire (nébuliseur, } \\
\text { humidificateur, opercule de fibroscopie) } \\
\text { - Perte de pression du ballonnet } \\
\text { - Drainage d'un pneumothorax }\end{array}$ & $\begin{array}{l}\text { - Déconnexion d'un élément du circuit } \\
\text { ventilatoire } \\
\text { (nébuliseur, humidificateur, opercule } \\
\text { de fibroscopie), } \\
\text { - Perte de pression du ballonnet } \\
\text { - Drainage d'un pneumothorax }\end{array}$ \\
\hline \multicolumn{3}{|c|}{$\begin{array}{l}\text { APRV : airway pressure release ventilation; BiPAP : bi-level positive airway pressure ; FR : fréquence respiratoire ; PEP : pression } \\
\text { expiratoire positive ; Pplat : pression de plateau ; Pcrête : pression de crête ; SDRA : syndrome de détresse respiratoire aiguë ; Ti : } \\
\text { temps inspiratoire ; Te : temps expiratoire ; VE : ventilation par minute (ou volume par minute) ; VSai : ventilation spontanée } \\
\text { avec aide inspiratoire ; Vte : volume courant expiratoire ; Vti : volume courant inspiratoire. }\end{array}$} \\
\hline
\end{tabular}

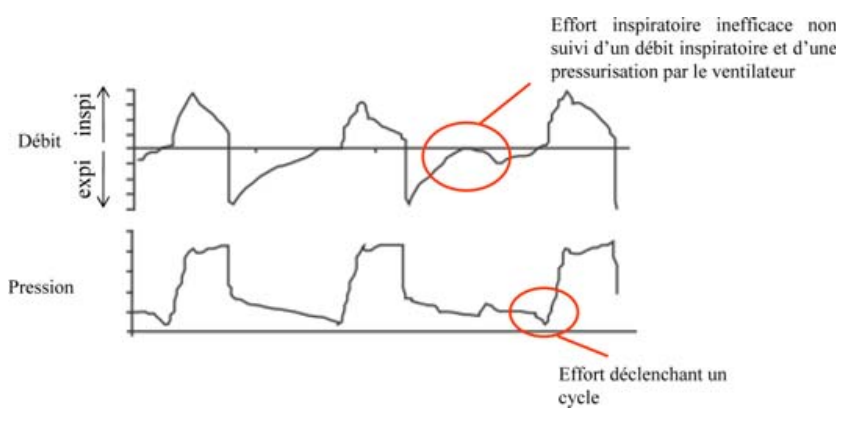

Fig. 4 Les efforts inefficaces sont des efforts inspiratoires non suivis du déclenchement d'un cycle respiratoire Inspi : inspiratoire ; expi : expiratoire

trigger pas assez sensible ou une surassistance ventilatoire avec un Vt trop élevé [19]. Leur conséquence principale est une fatigue ventilatoire avec une FR affichée par le ventilateur faussement basse et potentiellement faussement rassurante pour le soignant. En effet, le monitorage de la FR sur le ventilateur compte uniquement les cycles déclenchés et ne tient pas compte des efforts inefficaces. La FR réelle est donc la FR indiquée par le ventilateur à laquelle s'ajoutent les efforts inefficaces.

Le double-déclenchement est le déclenchement de deux cycles successifs sans expiration. Visible surtout en VAC, il est favorisé par un temps inspiratoire réglé trop court, un $\mathrm{Vt}$ trop bas, ou une demande inspiratoire importante du patient [20]. Sa conséquence principale est une augmentation importante de la pression intra-alvéolaire, puisque deux cycles successifs sont insufflés sans être expirés.

Les autodéclenchements sont des cycles délivrés par le ventilateur sans que le patient ne fasse d'effort inspiratoire. Ils peuvent être liés à un réglage trop sensible du trigger ou à une fuite sur le circuit, interprétée comme un effort inspiratoire par le ventilateur [19,20].

Des résistances pulmonaires élevées (bronchopneumopathies chroniques obstructives, asthme) et/ou une FR excessive peuvent être un obstacle à une expiration complète. Si l'expiration est incomplète, un volume sera trappé dans les poumons à chaque fin d'expiration. Ce volume accumulé est responsable de l'augmentation de la pression thoracique, ce qui favorise la survenue d'incidents hémodynamiques. Ce phénomène, appelé « auto-PEP » ou hyperinflation 
dynamique, n'est pas directement visualisable sur les courbes du ventilateur. Toutefois, un débit expiratoire persistant en fin d'expiration peut faire supposer que l'expiration est incomplète et que du volume s'accumule dans les poumons. La confirmation de la présence d'une hyperinflation dynamique se fera grâce à une pause téléexpiratoire, qui permettra, chez le patient sans effort respiratoire, de mesurer la pression réelle qui règne dans les poumons en fin d'expiration appelée PEP totale (auto-PEP $=$ PEP totale - PEP réglée) [23]. La présence d'une auto-PEP peut être responsable d'efforts inefficaces, car l'effort pour générer une pression négative et déclencher le ventilateur est plus élevé. Schématiquement lorsque l'auto-PEP est à $12 \mathrm{cmH}_{2} \mathrm{O}$ et que la PEP réglée sur le ventilateur est à zéro, le patient doit générer au minimum $-12 \mathrm{cmH}_{2} \mathrm{O}$ de force inspiratoire pour déclencher le ventilateur.

La ventilation mécanique est fréquemment source d'inconfort, qui peut participer à la désadaptation du patient [24]. La communication difficile et la limitation des mouvements peuvent jouer un rôle favorisant l'inconfort. Il peut être provoqué par les dispositifs de soins comme la sonde d'intubation et son système de fixation. L'inconfort peut également être lié à la dyspnée. Dans une étude récente, près de la moitié des patients interrogés signalaient une dyspnée sous ventilation mécanique qui pouvait être améliorée après ajustement des paramètres ventilatoires chez environ un tiers des patients [24]. Les réglages de la ventilation peuvent donc être source d'inconfort. L'évaluation systématique de la douleur avec les outils disponibles dans le service (échelle visuelle analogique [EVA], échelle comportementale...) contribue à limiter le vécu traumatisant de la réanimation. Tout comme la douleur et les constantes habituelles, la sensation de dyspnée devrait donc certainement être évaluée systématiquement chez le patient intubé éveillé en essayant de connaître les caractéristiques précises de celle-ci : impression de "manquer d'air ", impression que le ventilateur « pousse trop fort », impression de « forcer pour expirer ».

\section{Surveillance quotidienne des critères de sevrage}

Il y a un lien étroit entre la durée de ventilation mécanique et ses complications. Le moyen le plus efficace de les prévenir est un sevrage précoce de la ventilation mécanique invasive. L'évaluation quotidienne et systématique de critères de sevrage par les infirmiers(ières) permet de diminuer la durée de ventilation, et donc ses complications [25-27]. Ces critères portent sur l'état général et respiratoire du patient. Les critères généraux sont l'absence de vasopresseurs et d'inotropes, l'absence de sédation et une réponse cohérente aux ordres simples. Les critères respiratoires sont une $\mathrm{FiO}_{2} \leq 50 \%$ et une PEP $\leq 5 \mathrm{cmH}_{2} \mathrm{O}$. La toux efficace est également un critère du succès de l'extubation [27].

\section{Conclusion}

Une surveillance adaptée du patient nécessitant une ventilation artificielle invasive est indispensable à la prévention des complications de cette technique. La multiplicité des modes ventilatoires nécessite une adaptation des paramètres de surveillance. Si les aspects techniques de la ventilation doivent être maitrisés par les soignants, ils laissent entière l'importance de la communication avec le patient et l'optimisation de son confort.

Conflit d'intérêt : les auteurs déclarent ne pas avoir de conflit d'intérêt.

\section{Références}

1. Sole ML, Penoyer DA, Su X, et al (2009) Assessment of endotracheal cuff pressure by continuous monitoring: a pilot study. Am J Crit Care 18(2):133-43

2. SFAR, SRLF (2010) $5^{\mathrm{e}}$ Conférence de consensus. Prévention des infections nosocomiales en réanimation. Réanimation 19:4-14

3. Jaber S, El Kamel M, Chanques G, et al (2007) Endotracheal tube cuff pressure in intensive care unit: the need for pressure monitoring. Intensive Care Med 33(5):917-8

4. Windsor HM, Shanahan MX, Cherian K, et al (1976) Tracheal injury following prolonged intubation. Aust N Z J Surg 46 (1):18-25

5. Mercat A, Teboul JL (2008) Conséquences hémodynamiques de la ventilation mécanique. In: Brochard L, Mercat A, Richard JCM (eds) Ventilation artificielle, de la physiologie à la pratique. Ed Masson, Paris, pp 69-74

6. Mekontso-Dessap A (2008) Sevrage de la ventilation mécanique et système cardiovasculaire. In: Brochard L, Mercat A, Richard JCM (eds) Ventilation artificielle, de la physiologie à la pratique. Ed Masson, Paris, pp 236-42

7. Sauder P, Andreoletti M, Cambonie G, et al (2008) Sédation analgésie en réanimation (nouveau-né exclu). In: SFAR-SRLF, Conférence de consensus commune en réanimation. Réanimation 27(7-8):541-55

8. Orozco-Levi M, Torres A, Ferrer M, et al (1995) Semirecumbent position protects from pulmonary aspiration but not completely from gastroesophageal reflux in mechanically ventilated patients. Am J Respir Crit Care Med 152:1387-90

9. Torres A, Serra-Batlles J, Ros E, et al (1992) Pulmonary aspiration of gastric contents in patients receiving mechanical ventilation: the effect of body position. Ann Intern Med 116(7):540-3

10. Thille A, Cabello B, Brochard L (2009) Comment accélérer le sevrage de la ventilation mécanique ? Intérêt des modes ventilatoires, $37^{\mathrm{e}}$ Congrès de la SRLF, Elsevier Masson

11. Quenot JP, Ladoire S, Devoucoux F, et al (2007) Effect of a nurse-implemented sedation protocol on the incidence of ventilator-associated pneumonia. Crit Care Med 35:2031-6

12. Kress JP (2009) Clinical trials of early mobilization of critically ill patients. Supplement, ICU-acquired weakness: proceedings of a round table conference in Brussels. Crit Care Med 37:S442-S7 
13. Pohlman MC, Schweickert WD, Pohlman AS, et al (2010) Feasibility of physical and occupational therapy beginning from initiation of mechanical ventilation. Crit Care Med 38:2089-94

14. SFAR-SRLF (2009) $6^{\text {e }}$ Conférence de consensus. Mieux vivre la réanimation. Réanimation 19(3):191-203

15. Mercat A (2008) Échanges gazeux en ventilation mécanique. In: Brochard L, Mercat A, Richard JCM (eds) Ventilation artificielle, de la physiologie à la pratique. Ed Masson, Paris, pp 83-4

16. Ioos V, Galbois A, Chalumeau-Lemoine L, et al (2011) An integrated approach for prescribing fewer chest X-rays in the ICU. Ann Intensive Care 1(1):4

17. Richard JC, Girault C, Leteurtre S, et al (2005) Prise en charge ventilatoire du syndrome de détresse respiratoire aiguë de l'adulte et de l'enfant (nouveau-né exclu) — recommandations d'experts de la Société de réanimation de langue française. Réanimation 14:313-22

18. Mercat A (2008) Les modes ventilatoires conventionnels et mixtes. In: Brochard L, Mercat A, Richard JCM (eds) Ventilation artificielle, de la physiologie à la pratique. Ed Masson, Paris, pp $32-4$

19. Thille AW, Rodriguez P, Cabello B, et al (2006) Patient ventilator asynchrony during mechanical ventilation. Intensive Care Med $32: 515-22$

20. Thille AW (2008) Asynchronies patient-ventilateur: diagnostic et traitement. In: Brochard L, Mercat A, Richard JCM (eds) Venti- lation artificielle, de la physiologie à la pratique. Ed Masson, Paris, pp 85-93

21. Bosma K, Ferreyra G, Ambrogio C, et al (2007) Patientventilator interaction and sleep in mechanically ventilated patients: pressure support ventilation versus proportionnal assist ventilation. Crit Care Med 35:1048-54

22. Nilsestuen JO, Hargett KD (2005) Using ventilator graphics to identify patient-ventilator asynchrony. Respir Care 50:202-34; discussion 232-4

23. Jaber S, Sebbane M, Chanques G, et al (2006) Positive end expiratory pressure and intrinsic positive end expiratory pressure in assist ventilatory modes. Reanimation 5:81-6

24. Schmidt M, Demoule A, Polito A, et al (2011) Dyspnea in mechanically ventilated critically ill patients. Crit Care Med 39 (9):2059-65

25. Ely EW, Baker AM, Dunagan DP, et al (1996) Effect on the duration of mechanical ventilation identifying patients capable of breathing spontaneously. N Engl J Med 19 335(25):1864-9

26. SRLF (2001) $21^{\mathrm{e}}$ Conférence de consensus, sevrage de la ventilation mécanique (à l'exclusion du nouveau-né et du réveil d'anesthésie)

27. Blackwood B, Alderdice F, Burns K, et al (2011) Use of weaning protocols for reducing of mechanical ventilation in critically ill adult patients: Cochrane systematic review and meta-analysis. BMJ 342:c7237 